This is an author-created version of an article accepted for publication in Current Medical Imaging Reviews (Curr Med Imag Rev), vol. 9, no. 2, pp. 96-101, 2013. DOI: 10.2174/1573405611309020004

\title{
C-arm CT Perfusion Imaging in the Interventional Suite
}

\author{
Andreas Fieselmann ${ }^{1,2,3}$ and Michael Manhart ${ }^{1,3}$ \\ ${ }^{1}$ Department of Computer Science, Pattern Recognition Lab, Friedrich-Alexander \\ University of Erlangen-Nuremberg, Erlangen, Germany \\ ${ }^{2}$ Erlangen Graduate School in Advanced Optical Technologies (SAOT), Erlangen, \\ Germany \\ ${ }^{3}$ Siemens AG, Healthcare Sector, Erlangen / Forchheim, Germany \\ E-mail: andreas.fieselmann@siemens.com, \\ michael.manhart@informatik.uni-erlangen.de
}

\begin{abstract}
Diagnostic perfusion imaging using CT or MRI has been available for several years. One of its applications is acute stroke diagnosis. Interventional perfusion imaging using $\mathrm{C}$-arm CT is a novel field of research. It could provide perfusion information during catheter-guided stroke treatment in order to optimize patient management. In this survey article, the clinical and technical background of this topic are described and first results from in-vivo measurements are presented.
\end{abstract}

Keywords: C-arm CT, interventional imaging, perfusion, stroke, survey 


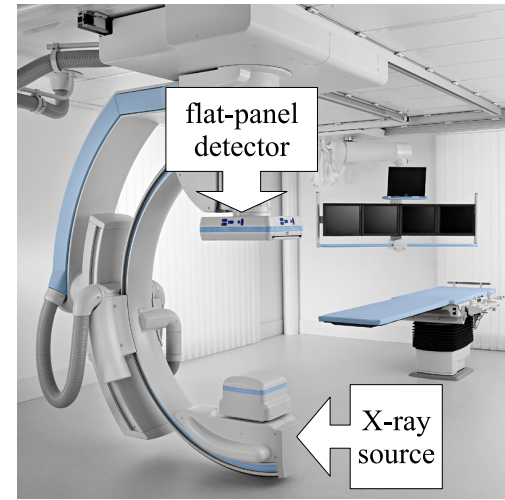

(a) monoplane system (Artis zee ceiling-mounted)

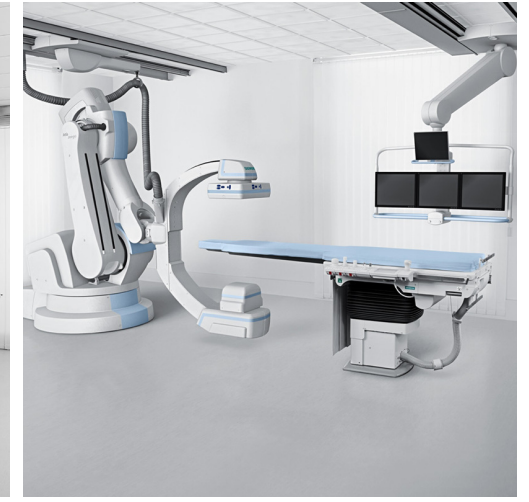

(b) robotic, monoplane system (Artis zeego)

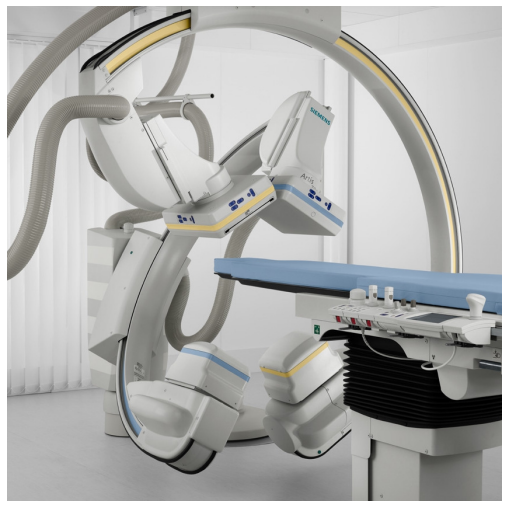

(c) biplane system

(Artis zee biplane)

Figure 1. Different C-arm angiography systems (Siemens AG, Healthcare Sector, Forchheim, Germany) capable of CT-like imaging

\section{Introduction}

Interventional perfusion imaging using $\mathrm{C}$-arm CT is a novel field of research [1]. In this introductory section, the clinical background and motivation as well as the technical challenges of this approach will be described.

\subsection{Perfusion Imaging and Stroke Treatment}

Acute stroke is a serious medical emergency. In an ischemic stroke event the blood supply to the brain is locally disrupted. Perfusion CT and MRI can support stroke diagnosis. In order to obtain perfusion images the basic workflow is the same for both modalities. A contrast agent is injected and images of the brain are acquired at short, typically $0.5-1 \mathrm{~s}$, intervals over a period of about $40 \mathrm{~s}$.

Time-attenuation curves (TAC) can be measured which describe the local change of $\mathrm{X}$-ray attenuation due to the inflow and outflow of contrast agent. Perfusion parameters such as cerebral blood flow (CBF) and volume (CBV), mean transit time (MTT) and time-to-peak (TTP) can be computed from these curves [2]. These parameters are usually displayed to the physician using color-coded 2-D parameter maps. They can be used to identify tissue that would benefit from stroke treatment.

There are several options for stroke treatment. For example, depending on the time after stroke onset, a pharmaceutical can be injected intra-venously (intravenous thrombolysis) or can be administered locally by a catheter (intra-arterial thrombolysis) [3. This pharamaceutical dissolves the clot causing an ischemic stroke. A further treatment option is mechanical thrombolysis where a catheter removes the clot mechanically.

Catheter-guided stroke treatment is usually carried out in the interventional suite with help of a C-arm angiography system (Figure1). A C-arm system is used to navigate the catheter to the stroke site using acquired 2-D X-ray images. While 2-D imaging 
is the main application of a $\mathrm{C}$-arm angiography system during stroke treatment, 3-D tomographic perfusion imaging could be very helpful. The next section will discuss the advantages when a C-arm angiography system could be used for tomographic perfusion imaging.

\subsection{Enhancing Stroke Treatment with Perfusion C-arm CT}

For several years it is possible to obtain reconstructed 3-D volumes from projections acquired with a C-arm angiography system [4. This modality, also known as C-arm CT or 3-D rotational angiography, could be used to acquire perfusion information directly in the interventional suite. Below we list three potential advantages of perfusion imaging using C-arm CT.

(i) It could be possible to acquire a perfusion scan immediately before starting the treatment in order to re-asses the state of perfusion. Note that a significant amount of time may be needed in order to relocate the patient [5] after an initial perfusion CT or MR in which the state of perfusion may have changed.

(ii) Perfusion imaging during the procedure could be used to determine the treatment success and treatment endpoint. This could make catheter-guided stroke treatment more effective.

(iii) A new workflow could be used for patients that are suspected to have a stroke. The initial diagnostic perfusion exam is carried out in the interventional suite. If the patient is selected for catheter-guided stroke treatment then no further patient relocation is necessary. This would accelerate stroke treatment. Note that the average stroke patient loses 1.9 million neurons per minute [6] and fast stroke treatment is mandatory.

\subsection{Technical Challenges}

Perfusion C-arm CT has certain technical challenges. They are related to the low temporal sampling rate and the low temporal resolution of 3-D volumes acquired using C-arm CT. When using C-arm CT for time-resolved tomographic imaging, several consecutive rotations of the $\mathrm{C}$-arm are necessary which are performed in alternating directions of rotation. With standard reconstruction algorithms such as Feldkamp-type algorithms [7] one 3-D volume can be reconstructed from the projection data of one short-scan covering an angular range of approximately $200^{\circ}$.

The comparably low C-arm rotation speed (typically 4-6 s per 200 ) [4] and the time interval needed between two alternating $\mathrm{C}$-arm rotations (typically $1 \mathrm{~s}$ ) limits the temporal sampling of the dynamic processes, i.e. the flow of the contrast agent in the

brain tissue. For comparison, recall that the sample period in perfusion CT and MR is usually $0.5-1 \mathrm{~s}$. Longer sample periods can lead to an undersampling of the TACs which constitutes the major technical challenge in perfusion C-arm CT. Perfusion parameters may not be computed correctly from these undersampled data. 
The temporal resolution is inversely proportional to the time needed to acquire one full set of projection data (e.g. $200^{\circ}$ angular range). Since the projection data are acquired over a comparably long time interval these data are actually inconsistent. With common static reconstruction algorithms artifacts can arise due to time-varying attenuation values [8]. These reconstruction artifacts may lead to incorrect perfusion data.

\section{Literature Survey}

The techniques proposed to deal with the challenges of perfusion C-arm CT can be divided into three classes: dynamic analytical reconstruction algorithms, dynamic iterative reconstruction algorithms and novel C-arm systems with higher acquisition speed.

The analytical approaches are based on filtered backprojection (FBP) and can be implemented such that they are computationally very fast [9]. Montes and Lauritsch presented an analytical method for perfusion imaging with a slower continuously rotating CT scanner based on a dynamic FBP reconstruction algorithm [10. The motivation for this method was to reduce X-ray dose by decreasing the amount of acquired projection data when operating the CT scanner at a low rotation speed. An extension of this approach for C-arm CT was introduced by Fieselmann et al. [11. It is termed the IS-PRI (interleaved scanning-partial reconstruction interpolation) method and uses several, interleaved C-arm CT acquisitions to increase the temporal sampling and resolution of the reconstructed TACs. An introduction to IS-PRI is given in Section 3.1. Neukirchen 12 presented an adaption of the approach by Montes and Lauritsch to data acquired using bi-directional $\mathrm{C}$-arm rotations by interpolating the missing projection data individually for each view angle by least-squares curve fitting with Fourier basis functions.

The iterative approaches are based on algebraic reconstruction techniques (ART) [13], which are computationally much more demanding than analytical methods. However, they allow to incorporate prior knowledge about the shape of the reconstructed TACs and the possibility for noise reduction by non-linear regularization of the reconstruction. Serowy et al. [14] and Neukirchen et al. [15] showed iterative modelbased approaches which reconstruct TACs described by a sum of weighted smooth temporal basis functions. Recently, Manhart et al. [16] combined this approach with non-linear filtering for noise reduction. This method is discussed in more detail in Section 3.3. Another technique was introduced by Beister et al. [17], where TAC samples are reconstructed from limited angle scans and the missing angular range is compensated by additional information from a prior image. This approach is similar to the temporal resolution improvement method based on prior image constrained compressed sensing (PICCS) for cardiac CT imaging by Chen et al. [18], which could potentially also be applied for perfusion C-arm CT.

A further option is to use novel C-arm systems with rapid scanning speed up to 


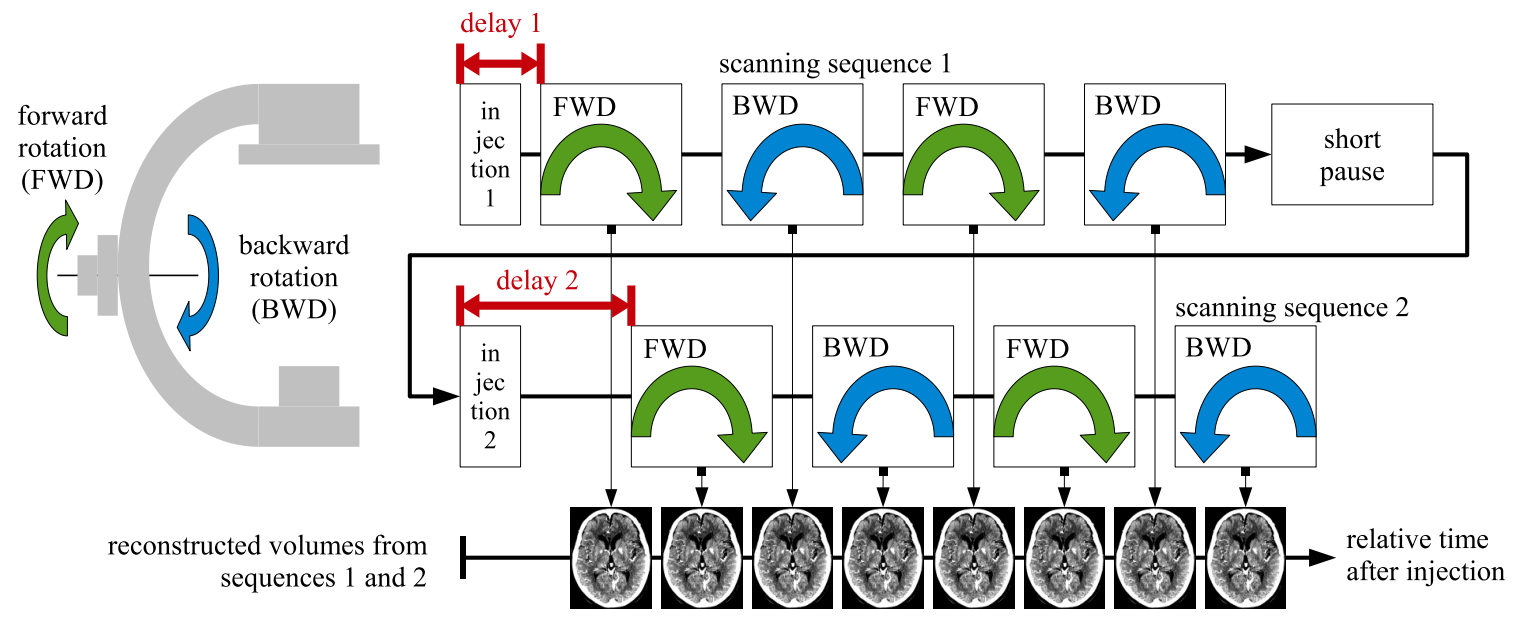

Figure 2. Illustration of an interleaved scanning protocol with $N_{\text {seq }}=2$ interleaved sequences each containing $N_{\text {rot }}=4$ C-arm rotations.

$100^{\circ}$ s. Recently, promising results from studies based on simulations [19] and animal scans [20] with an experimental high-speed acquisition protocol have been presented. More details on this possibility are given in Section 3.2.

Imaging of static perfusion (CBV) with C-arm CT has also been demonstrated and validated using a two-scan protocol [21, 22].

\section{Technical Approaches and In-vivo Measurements}

In this section, three technical approaches for C-arm CT perfusion imaging will be described and first results from in-vivo measurements will be presented.

\subsection{Interleaved Scanning and Partial Reconstruction Interpolation}

The interleaved scanning and partial reconstruction interpolation approach (IS-PRI) is a combination of a novel acquisition protocol and a dedicated reconstruction technique [1, 11, 23, 24]. The aim is to increase temporal sampling with interleaved scanning (IS) and compensate for the inconsistencies in the acquired data with partial reconstruction interpolation (PRI).

The basic idea of the interleaved scanning acquisition protocol is to conduct $N_{\text {seq }}$ scanning sequences (typically $N_{\text {seq }} \in[2,3]$ ) each with a new, identical contrast bolus injection. One scan sequence consists of $N_{\text {rot }}$ individual scans (typically $N_{\text {rot }} \in[6,7]$ ) acquired using alternating directions of rotation of the C-arm. Each scan sequence has a different time delay between the start of injection and the start of scanning. Assuming similar contrast agent bolus flow during each scan sequences, this method allows to artificially increase temporal sampling by retrospectively combining the data of all scan 


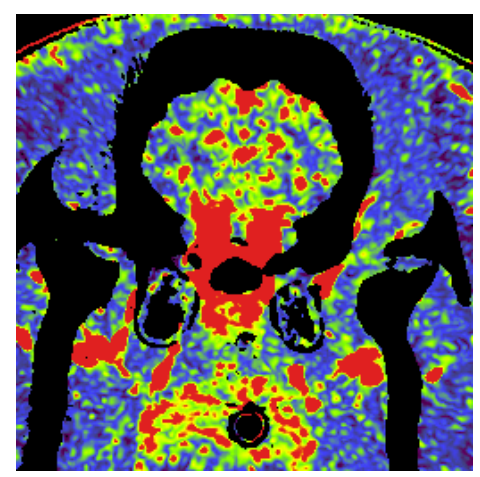

(a) coronal

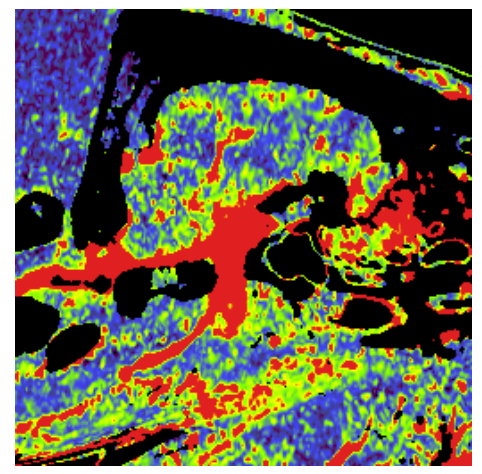

(b) sagittal

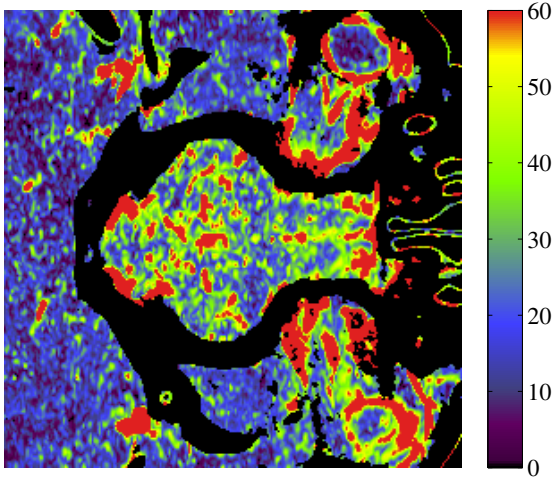

(c) transversal

Figure 3. Volume CBF maps (unit: $\mathrm{ml} / 100 \mathrm{~g} / \mathrm{min}$ ) of a perfusion-normal pig obtained using the interleaved scanning and partial reconstruction interpolation approach [1]. Images acquired at the Axiom lab, Dept. of Radiology, Stanford University.

sequences. The different time delays enable to measure different time points of the contrast agent flow in the brain tissue. Figure 2 illustrates this method with $N_{\text {seq }}=2$ and $N_{\text {rot }}=4$ (for simplicity, only 4 scans per sequence are displayed).

As already mentioned in Section 1.3, the projection data of one C-arm rotation is inconsistent due to the intentional contrast agent flow. Partial reconstruction interpolation is applied to reduce the effect of these inconsistencies. The full angular range of one $\mathrm{C}$-arm rotation (e.g. $200^{\circ}$ ) is subdivided into smaller angular intervals (e.g. $200^{\circ} / 6 \approx 33^{\circ}$ ). The projection data from these smaller angular intervals is individually reconstructed using an analytical Feldkamp-type algorithm [7]. The result is a set of partial reconstructions of 3 -D volumes corresponding to different time points in the scanning sequence.

A complete $3-\mathrm{D}$ volume, corresponding to a certain time point $t_{\mathrm{rec}}$, is obtained by summing up the partial reconstructions of all angular intervals (e.g. $0^{\circ}-33^{\circ}, 33^{\circ}-66^{\circ}$, ...) that also correspond to $t_{\mathrm{rec}}$. For each angular interval, temporal interpolation of the partial reconstruction is used in order to obtain the partial reconstruction for $t_{\text {rec }}$. Note that the data from the interleaved scanning sequences can be combined to yield more accurate interpolation results. In general, temporal interpolation is possible since time-attenuation curves are slowly varying functions. Since the reconstruction is applied to data acquired in a shorter time interval (e.g. 1/6 of the duration of one full C-arm rotation), the inconsistencies in the data are less prominent and reconstruction artifacts are reduced compared to a standard reconstruction.

Figure 3 shows CBF results obtained using the IS-PRI approach. Three orthogonal slices of the brain of a perfusion-normal pig are presented. Details about the scan and injection protocol can be found in [1]. Note that the coronal and transversal CBF maps are symmetrical as it is expected from a healthy animal.

The IS-PRI approach can be used with current C-arm CT systems having C-arm rotation times of 4-6 s. As clinically required, the image reconstruction is 
computationally fast since it is based on analytical reconstruction algorithms and can be carried out in a short time. Results from clinical studies suggest that already two interleaved scan sequences can enable reliable tissue perfusion measurement using the IS-PRI approach [1, 23].

\subsection{Rapid Scanning}

Another interesting possibility to enable perfusion C-arm CT is rapid scanning with novel C-arm systems allowing increased rotation speed (Artis zeego, Siemens AG, Forchheim, Germany; Figure 1(b). Such an experimental high-speed acquisition protocol has been evaluated in recent studies. Using this protocol each sweep acquires 133 projections in a $200^{\circ}$ angular range and requires $T_{\mathrm{r}}=2.8 \mathrm{~s}$ for data acquisition and a pause of $T_{\mathrm{w}}=1.2 \mathrm{~s}$ between sweeps. Thus TACs can be sampled with a temporal resolution of $T_{\mathrm{s}}=T_{\mathrm{r}}+T_{\mathrm{w}}=4 \mathrm{~s}$. However, using FBP-type reconstruction algorithms the limited number of 133 projections leads to streak artifacts and increased noise level. Manhart et al. [19] used an iterative algorithm applying a wavelet-based regularizer to reduce noise and streak artifacts in a simulation study with a digital brain perfusion phantom. Royalty et al. [20] used the rapid scanning protocol in an animal study with a canine stroke model. Examples for reconstructed CBF maps using this technique are shown in Figure 4 in comparison with maps acquired with PCT as gold standard.

\subsection{Iterative Image Reconstruction}

Dynamic iterative reconstruction algorithms are an interesting field of study. They allow to improve the temporal resolution of the reconstructed TACs by incorporating prior knowledge about the dynamic behavior of the TACs, e.g. by modeling the TACs as a weighted sum of spline basis functions. Furthermore, they offer possibilities for noise reduction using non-linear regularizers. Manhart et al. [16] used a dynamic iterative reconstruction (DIR) approach describing the TACs by a weighted sum of linear spline functions to calculate perfusion maps from a $\mathrm{C}$-arm acquisition protocol requiring $T_{\mathrm{r}}=4.3 \mathrm{~s}$ for data acquisition and a pause of $T_{\mathrm{w}}=1.2 \mathrm{~s}$ between the sweeps. A regularization based on bilateral filtering is used to reduce noise and increase CNR in the tissue TACs. Figure 4 shows perfusion maps reconstructed with DIR from a canine animal study compared to the RS results and PCT maps as gold standard.

\section{Summary and Outlook}

Pre- and intra-procedural perfusion imaging could significantly enhance catheterguided stroke treatment. A C-arm CT system can be used to acquire time-resolved reconstructed volumes after contrast agent injection from which perfusion information is computed. However, the C-arm rotation speed of currently available systems is comparably low which limits the temporal sampling. Perfusion C-arm CT imaging is still a challenging task and it is not commercially available yet. 


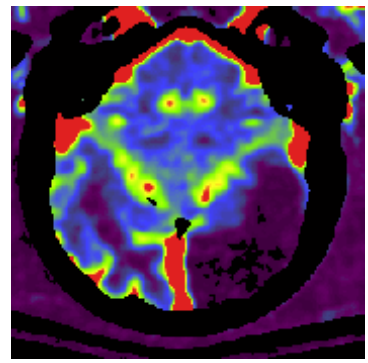

(a) PCT, slice 1

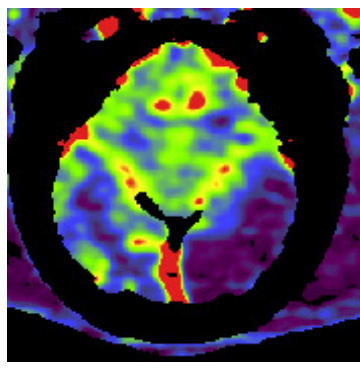

(d) RS, slice 1

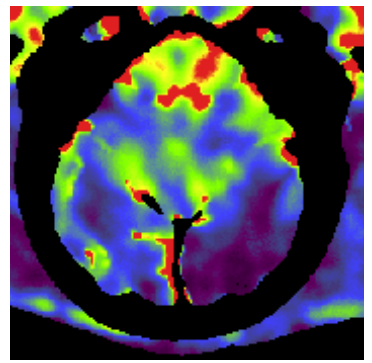

(g) DIR, slice 1

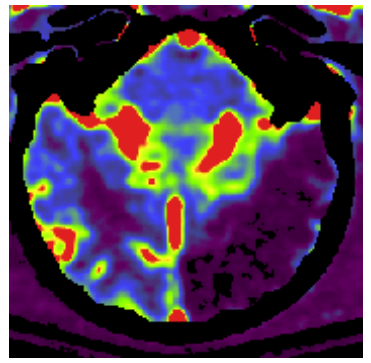

(b) PCT, slice 2

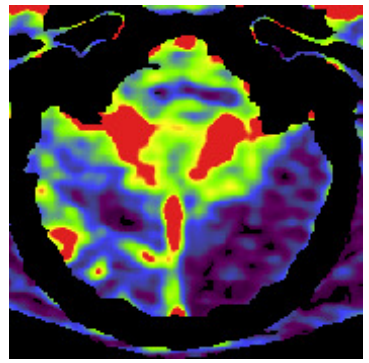

(e) RS, slice 2

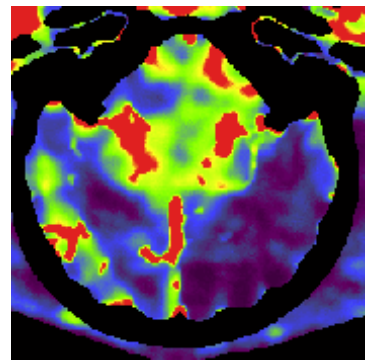

(h) DIR, slice 2

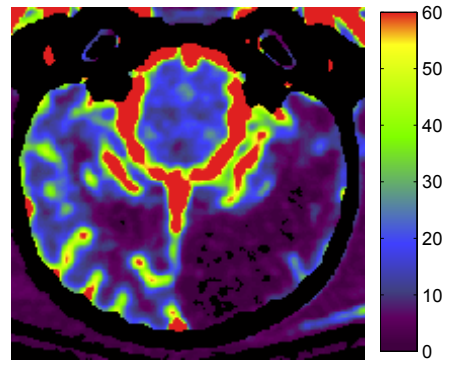

(c) PCT, slice 3

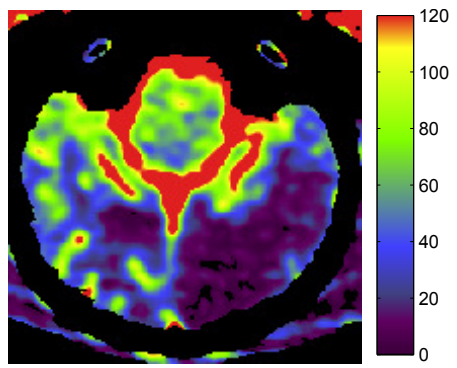

(f) RS, slice 3

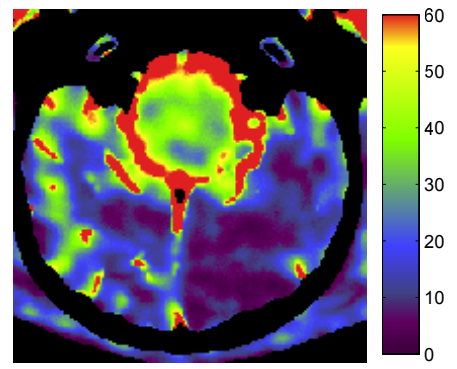

(i) DIR, slice 3

Figure 4. $(\mathrm{a}-\mathrm{c}$ ) CBF maps (units: $\mathrm{ml} / 100 \mathrm{~g} / \mathrm{min}$ ) of a canine with an induced ischemic stroke obtained with perfusion CT (PCT). (d-f) perfusion C-arm CT (PCCT) using the rapid scanning (RS) approach [19]. (g-i) PCCT using the dynamic iterative reconstruction approach (DIR) [16]. The 3-D volume CBF maps from PCCT were registered onto the 2-D maps from PCT. Images acquired by Dr. C. Strother, Dept. of Radiology, University of Wisconsin-Madison.

To date, only few methods have been presented in the literature regarding this novel research topic. Dynamic analytical and iterative reconstruction algorithms have been proposed in order to compensate the low C-arm rotation speed. Furthermore, investigations were carried out using novel C-arm systems with higher acquisition speed.

Results from in-vivo measurements show very promising correlations of perfusion C-arm CT with perfusion CT. Clinical patient studies are necessary in order to further validate the current approaches under realistic conditions.

Perfusion C-arm CT is an ongoing topic of research. In the future, new image reconstruction approaches or new $\mathrm{C}$-arm CT scanning concepts could be investigated. Interleaved scanning using biplane angiography systems (Figure 1(c) could offer certain advantages [11]. Perfusion imaging with continuously-rotating C-arm CT systems could 
enable to use new concepts such as sliding-window reconstruction [8]. C-arm CT systems with fast $\mathrm{C}$-arm rotation speed are a promising option for interventional perfusion imaging that could be investigated in more detail.

\section{Conflict of Interest}

Andreas Fieselmann is an employee of Siemens AG and Michael Manhart receives financial support by Siemens AG.

\section{Acknowledgments and Disclaimer}

The authors gratefully acknowledge funding of the Erlangen Graduate School in Advanced Optical Technologies (SAOT) by the German Research Foundation (DFG) in the framework of the German excellence initiative. The concepts and information presented in Section 3 of this paper are based on research and are not commercially available.

\section{References}

[1] Fieselmann A. Interventional Perfusion Imaging Using C-arm Computed Tomography: Algorithms and Clinical Evaluation. University of Erlangen-Nuremberg, Germany; 2011.

[2] Fieselmann A, Kowarschik M, Ganguly A, Hornegger J, Fahrig R. Deconvolution-Based CT and MR Brain Perfusion Measurement: Theoretical Model Revisited and Practical Implementation Details. Int J Biomedical Imaging. 2011;2011, article ID 467563, 20 pages.

[3] González RG, Hirsch JA, Koroshetz WJ, Lev MH, Schaefer PW, editors. Acute Ischemic Stroke. 1st ed. Berlin, Germany: Springer; 2006.

[4] Strobel N, Meissner O, Boese J, et al . 3D Imaging with Flat-Detector C-Arm Systems. In: Reiser MF, Becker CR, Nikolaou K, Glazer G, editors. Multislice CT. 3rd ed. Berlin, Germany: Springer; 2009. p. 33-51.

[5] Nedeltchev K, Arnold M, Brekenfeld C, et al . Pre- and In-Hospital Delays From Stroke Onset to Intra-arterial Thrombolysis. Stroke. 2003;34(5):1230-1234.

[6] Saver JL. Time Is Brain - Quantified. Stroke. 2006;37(1):263-266.

[7] Feldkamp LA, Davis LC, Kress JW. Practical cone-beam algorithm. J Opt Soc Am. 1984;A1:612619.

[8] Fieselmann A, Dennerlein F, Deuerling-Zheng Y, Boese J, Fahrig R, Hornegger J. A model for filtered backprojection reconstruction artifacts due to time-varying attenuation values in perfusion C-arm CT. Phys Med Biol. 2011;56(12):3701-3717.

[9] Rohkohl C, Keck B, Hofmann H, Hornegger J. RabbitCT - an open platform for benchmarking 3D cone-beam reconstruction algorithms. Med Phys. 2009;36(9):3940-3944.

[10] Montes P, Lauritsch G. A temporal interpolation approach for dynamic reconstruction in perfusion CT. Med Phys. 2007;34(7):3077-3092.

[11] Fieselmann A, Ganguly A, Deuerling-Zheng Y, et al . Interventional 4-D C-arm CT Perfusion Imaging Using Interleaved Scanning and Partial Reconstruction Interpolation. IEEE Trans Med Imaging. 2012;31(4):892-906.

[12] Neukirchen C. An extended temporal interpolation approach for dynamic object reconstruction. In: Proceedings of the International Meeting on Fully Three-Dimensional Image Reconstruction in Radiology and Nuclear Medicine 2011. Potsdam, Germany; 2011. p. 379-382. 
[13] Kak AC, Slaney M. Principles of Computerized Tomographic Imaging. 1st ed. New York, USA: IEEE Press; 1988.

[14] Serowy S, Gurvit O, Skalej M, Rose G. A Jacobi-like Solution to the Model Based Tomographic XRay Perfusion Imaging. In: Proceedings of the IEEE Nuclear Science Symposium and Medical Imaging Conference 2007. Hawaii, USA; 2007. p. 3085-3088.

[15] Neukirchen C, Giordano M, Wiesner S. An iterative method for tomographic x-ray perfusion estimation in a decomposition model-based approach. Med Phys. 2010;37(12):6125-6141.

[16] Manhart M, Kowarschik M, Fieselmann A, Deuerling-Zheng Y, Royalty K, Maier A, et al. Dynamic Iterative Reconstruction for Interventional 4-D C-Arm CT Perfusion Imaging. IEEE Trans Med Imaging. 2013; (in press).

[17] Beister M, Kyriakou Y, Kalender W. Increased Temporal Resolution for Dynamic Perfusion CT (PCT) with C-arm Flat-Detector CT. In: Proceedings of the Scientific Assembly and Annual Meeting of the RSNA 2010. Chicago, USA; 2010. .

[18] Chen GH, Tang J, Hsieh J. Temporal resolution improvement using PICCS in MDCT cardiac imaging. Med Phys. 2009;36(6):2130-2135.

[19] Manhart M, Fieselmann A, Deuerling-Zheng Y. Evaluation of a Tight Frame Reconstruction Algorithm for Perfusion C-arm CT Using a Realistic Dynamic Brain Phantom. In: Proceedings of the Second International Conference on Image Formation in X-Ray Computed Tomography. Salt Lake City, USA; 2012. p. 123-126.

[20] Royalty K, Manhart M, Pulfer K, Deuerling-Zheng Y, Strother C, Fieselmann A, et al. C-Arm CT Measurement of Cerebral Blood Volume and Cerebral Blood Flow Using a Novel High-Speed Acquisition and a Single Intravenous Contrast Injection. Am J Neuroradiol. 2013;(in press).

[21] Zellerhoff M, Deuerling-Zheng Y, Strother CM, et al . Measurement of cerebral blood volume using angiographic C-arm systems. In: Proceedings of SPIE Medical Imaging 2009: Biomedical Applications in Molecular, Structural, and Functional Imaging. vol. 7262. Lake Buena Vista, USA; 2009. p. 72620H1-8.

[22] Struffert T, Deuerling-Zheng Y, Kloska S, et al . Cerebral blood volume imaging by flat detector computed tomography in comparison to conventional multislice perfusion CT. Eur Radiol. 2011;21(4):882-889.

[23] Ganguly A, Fieselmann A, Marks M, et al . Cerebral CT Perfusion Using an Interventional C-arm Imaging System: Cerebral Blood Flow Measurements. Am J Neuroradiol. 2011;32(8):1525-1531.

[24] Ganguly A, Fieselmann A, Boese J, Rohkohl C, Hornegger J, Fahrig R. In vitro evaluation of the imaging accuracy of C-arm conebeam CT in cerebral perfusion imaging. Med Phys. 2012;39(11):6652-6659. 